\title{
Aetiology and Prognosis of Pancreatic Pseudo Cysts Over A 10 Year Period
}

\author{
Maged Farag*, Xavier Escofet and Nader Naguib \\ Department of General Surgery, Prince Charles Hospital, UK
}

*Corresponding author: Maged Farag, Department of General Surgery, Prince Charles Hospital, UK

\begin{abstract}
Introduction: Pancreatic pseudocysts often arise as a complication of acute or chronic pancreatitis with reported prevalence in chronic pancreatitis of 20-40\%. Most common cause is alcoholic chronic pancreatitis (70-78\%) then idiopathic chronic pancreatitis (6-16\%), then biliary pancreatitis (6-8\%). The aim of this study is to assess the demographic characteristic of patients diagnosed with pseudocysts, aetiology, characteristic features, and prognosis.

Methods: Prospective observational study to follow up patients diagnosed with pancreatic pseudocyst in 5 years (2006-2011). Data was collected in 2011 and patients followed up till June 2019. Radiology database searched for all cases that had a diagnosis confirmation by Computerised Tomography (CT). Total of 167 CT carried out for 119 patients. 35 patients were excluded due to absence of pancreatic pseudocyst after radiological re-evaluation.

Results: In 5 years, 84 patients diagnosed with pancreatic pseudocysts on CT. 127 CT scans done mainly for follow up. 51(60.7\%) males and $33(39.3 \%)$ females; mean age $=57.8$ years (20-93). 41 cases (48.8\%) were associated with acute pancreatitis $(8$ on background of chronic pancreatitis). 21 cases (25\%) were associated with chronic pancreatitis, 4 were associated with pancreatic malignancy while $18(21.4 \%)$ were reported as incidental finding. The underlying factor was alcoholic pancreatitis in 37 patients (44\%), gall stones in 19 patients (22.6\%), pancreatic malignancy in $4(4.8 \%)$ patients, biliary strictures in 2 patients and trauma in 2 patients. Idiopathic pancreatic pseudocysts were seen in 20 patients (23.8\%), 18 of them were incidentally found on CT scan. 8 cases ( $40 \%$ ) of idiopathic pseudocysts, were associated with non-pancreatic malignancies. 50\% mortality (42 patients). 17 had acute pancreatitis, 15 had chronic pancreatitis, 6 were from the incidental finding and 4 from acute on top of chronic group. As for aetiology, 25 patients of the 42 had alcoholic pancreatitis, 12 had gall stones pancreatitis, 3 were unknown aetiology and 2 had pancreatic cancer.

Conclusion: Our study showed that alcoholic pancreatitis remains the most frequent underlying aetiology for pancreatic pseudocysts although it is not as common as previously reported. Idiopathic pseudocysts constituted a substantial number of this study with a higher than expected incidental pseudocysts. The association of pancreatic pseudocysts with malignancy needs to be further evaluated.
\end{abstract}

\section{Introduction}

Pancreatic pseudo-cysts often arise as a complication of acute or chronic pancreatitis. Previous reported prevalence of pancreatic pseudo-cysts in chronic pancreatitis ranges from $20 \%$ to $40 \%$. Pancreatic pseudo-cysts most commonly arise in patients with alcoholic chronic pancreatitis $(70 \%$ to $78 \%)(1,2)$. The second most common cause is idiopathic chronic pancreatitis (6\% to $16 \%$ ), followed by biliary pancreatitis (6\% to $8 \%$ ) (3). Various imaging modalities are used in the diagnosis of pancreatic pseudo-cysts

with Computed Tomography (CT) being the gold standard with $82 \%$ to $100 \%$ sensitivity and $98 \%$ specificity (4). There is wide variability in the range of spontaneous regression in the literature from 8 to $70 \%$, with almost $40 \%$ of cysts that are less than 6 weeks old resolving without intervention compared to around $10 \%$ of the cysts older than 6 weeks (5). The aim of this study is to assess the demographic characteristic of diagnosed patients, aetiology of the pseudo-cysts, their characteristic features, prognosis and mortality rate. 


\section{Methods}

This is a prospective observational study to follow up all consecutive patients diagnosed with pancreatic pseudo-cyst over a period of five years (2006 - 2011) in a district general hospital. Data collected retrospectively in 2011 to identify patients with pseudopancreatic cysts. The search was conducted using the radiology database of all cases had a diagnostic confirmation of pseudo pancreatic cyst by Computerized Tomography (CT). A total number of 167 CT scans were carried out for 119 patients. Radiological re-evaluation was conducted by a designated radiologist. 35 patients were excluded from the study due to absence of pancreatic pseudo-cyst after radiological re-evaluation. The remaining 84 patients were followed up till June 2019. The following demographic data were collected (age, gender, aetiology of pancreatitis, blood tests including amylase, liver function tests, white cell count, serum calcium and oxygen saturation). Mode of treatment and related complications. Radiological characteristics of the pancreatic pseudo cyst included: site, size, duration, calcification within the cyst. Other parameters observed prospectively were; mortality/ morbidity, further episodes of pancreatitis and duration between diagnosis and mortality/ morbidity. Subgroup analysis was conducted to look at prevalence of different aetiologies in both genders. Statistical analysis was conducted using Fisher Exact test, Mann-Whitney U test and the multivariate analysis was carried out using SPSS version 25 for Windows (SPSS Inc, Chicago, IL, USA). P value $\leq .05$ was considered significant.

\section{Results}

In five years, period (2006 - 2011), 84 patients were diagnosed with pancreatic pseudo-cysts on CT scan. These patients had a total of 127 CT scans mainly for follow up. There were 51 male patients $(60.7 \%)$ and 33 female patients (39.3\%) with mean age of 57.8 years (20 - 93). In 41 cases (48.8\%) the pseudo-cysts were associated with acute pancreatitis ( 8 on background of chronic pancreatitis). 21 cases $(25 \%)$ were associated with chronic pancreatitis, 4 were associated with pancreatic malignancy while in 18 cases $(21.4 \%)$ there was no obvious history of pancreatitis and the diagnosis was reported as incidental finding.

The underlying factor was alcoholic pancreatitis in 37 patients (44\%), gall stones in 19 patients (22.6\%), pancreatic malignancy in $4(4.8 \%)$ patients, biliary strictures in 2 patients and trauma in 2 patients. Idiopathic pancreatic pseudo-cysts were seen in 20 patients $(23.8 \%), 18$ of them were incidentally found on the CT scan. Of note, in 8 cases (40\%) of the idiopathic pseudo-cysts, were associated with non-pancreatic malignancies. In a subgroup analysis, alcohol was the commonest aetiology in male patients 29/51 (57\%) while in female patients, incidental pseudo-cysts constituted $11 / 33$ (33.3\%), $\mathrm{P}=0.23$. Gall stones were the 2 nd most common aetiology in females $10 / 33$ (30.3\%). Nine patients had more than one pseudo-cyst. The size of the pseudo-cysts varied significantly in reporting from small to huge pseudo-cysts with extension into the left thigh in one case. The body of the pancreas was the most common site (33 pseudo-cysts) followed by the head of the pancreas with 28 , tail 27 , uncinate 4 , neck 2 , not specified 7, 1 junction between body and tail, 2 junction between head and body (Table 1). The majority were managed conservatively with two drained percutaneously and two drained endoscopically. The 84 patients were followed up till June 2019 (mean follow up of 10 years). This showed that 42 patients (50\%) died (male: female, 21:21), mean age of 61.8 (27-93). 9 patients ( $21 \%$ of the mortality) died from complications related to the pancreatitis or due to complications from the pseudo-cyst like infection or bleeding into the cyst, with one patient dying from respiratory failure following laparoscopic cholecystectomy for gall stones pancreatitis (Table 2). The 9 patients represent $10.7 \%$ cause-related mortality. 17 of those 42 patients ( $40 \%$ ) were from the acute pancreatitis group, 15 patients (36\%) from the chronic pancreatitis group, 6 patients (14\%) from the incidental finding group and 4 patients (10\%) from the acute on chronic group. As regards the aetiology, 25 patients of the $42(59.5 \%)$ were from the alcoholic pancreatitis group, 12 patients (28.5\%) from the gall stones pancreatitis group, 3 patients $(7 \%)$ from the unknown aetiology group and 2 patients (5\%) from the pancreatic cancer group, Table 2. Multivariate analysis (MANOVA) yielded a highly significant association between the aetiology of pancreatitis and death, $p=0.007$; however, there was no significant association between the mode of pancreatitis (acute or chronic) and death, $p=0.338$, Table 3 . Using death or alive dichotomy, chi square test confirmed the highly significant relationship between the aetiology of pancreatitis and death, $p=.000$. A two-way analysis of variance yielded a significant relationship between the aetiology of pancreatitis and its mode of onset (acute or chronic), $p=0.000$ (Table 3). The duration of time form diagnosis of pancreatitis/ pancreatic pseudo-cyst to death was quite variable ranging from 8 to 3809 days (median 1018 days) for the whole cohort of mortality patients. The range for the cause specific cohort was 10 to 1424 days (median 112 days), p-value is .04006. 12 patients (14\%) had further episodes of pancreatitis requiring admission to hospital. The outcome from the follow up of the pancreatic pseudo-cysts over the study period is shown in Table 4.

Table 1: Sites of the pseudo-cysts within the pancreas.

\begin{tabular}{|c|c|}
\hline Site & Number \\
\hline Body & 33 \\
\hline Head & 28 \\
\hline Tail & 27 \\
\hline Uncinate & 4 \\
\hline Neck & 2 \\
\hline Junctional & $3^{*}$ \\
\hline Not Specified & 7 \\
\hline
\end{tabular}

*1 in junction between body and tail, 2 in junction between head and body. 
Table 2: Demographics of mortality patients.

\begin{tabular}{|c|c|c|}
\hline \multirow{3}{*}{ Gender } & Males & 21 \\
\cline { 2 - 3 } & Females & 21 \\
\hline \multirow{3}{*}{ Aetiology } & Alcoholic & 25 \\
\cline { 2 - 3 } & Gall stones & 12 \\
\cline { 2 - 3 } & Cancer & 2 \\
\hline \multirow{3}{*}{ Mode of pancreatitis } & Incidental & 3 \\
\cline { 2 - 3 } & Acute & 17 \\
\cline { 2 - 3 } & Chronic & 15 \\
\cline { 2 - 3 } & Acute on top of Chronic & 6 \\
\hline
\end{tabular}

Table 3: Multivariate analysis.

\begin{tabular}{|c|c|c|c|c|}
\hline \multirow{3}{*}{ Gender } & df & F & Sig. \\
\cline { 2 - 5 } & Aetiology of Pancreatitis & 1 & 2.121 & 0.149 \\
\hline \multirow{2}{*}{ Age at CT } & $\begin{array}{c}\text { Mode of Pancreatitis } \\
\text { (Acute/ Chronic) }\end{array}$ & 1 & 0.707 & 0.403 \\
\cline { 2 - 5 } & Aetiology of Pancreatitis & 1 & 4.83 & 0.031 \\
\hline \multirow{3}{*}{ Meath } & $\begin{array}{c}\text { Mode of Pancreatitis } \\
\text { (Acute/ Chronic) }\end{array}$ & 1 & 0.273 & 0.603 \\
\cline { 2 - 5 } & $\begin{array}{c}\text { Aetiology of Pancreatitis } \\
\text { Mode of Pancreatitis } \\
\text { (Acute/ Chronic) }\end{array}$ & 1 & 7.556 & 0.007 \\
\hline
\end{tabular}

Table 4: Pseudo-cyst follow-up outcome (June 2019).

\begin{tabular}{|c|c|}
\hline Outcome & Number \\
\hline Resolution & $40(47.6 \%)$ \\
\hline No further imaging & $15(17.8 \%)$ \\
\hline Stable in size & $9(10.7 \%)$ \\
\hline Decreased in size & $6(7 \%)$ \\
\hline Patient death & $5(5.9 \%)$ \\
\hline Malignant & $3(3.5 \%)$ \\
\hline Increased in size & $2(2.3 \%)$ \\
\hline Drained & $1(1 \%)$ \\
\hline Infected & $1(1 \%)$ \\
\hline Surgery (Pancreatectomy) & $1(1 \%)$ \\
\hline Unknown & $1(1 \%)$ \\
\hline
\end{tabular}

\section{Discussion}

Pancreatic pseudocysts are the commonest pancreatic cystic lesions and represent 75\%-80\% [1]. They are localized fluid collections rich in amylase and other pancreatic enzymes that gets surround by fibrous tissue wall not lined by epithelium [2]. Pseudocysts are a common clinical problem and arise as a complication of chronic pancreatitis in up to $40 \%$ of cases [3]. Alcoholic pancreatitis is the most common cause and account for over $75 \%$ of cases in some series [4]. The incidence of pancreatic pseudocysts is low and ranges between $1.6 \%-4.5 \%$ irrespective of the aetiology $[5,6]$. The pathogenesis of pancreatic pseudocysts is the disruption to the pancreatic duct as a result of pancreatitis or trauma which results in extravasation of pancreatic secretions. Two thirds of patients with pseudocysts have demonstrable connections between the cyst and the pancreatic duct. In the other third, an inflammatory reaction most likely sealed the connection so that it is not demonstrable [7]. In acute pancreatitis, fluid collections persisting for more than 4-6 weeks that are lined by a well-defined wall of fibrous or granulation tissue, would be regarded as acute pseudocysts [2,4]. In chronic pancreatitis the mechanism is less clear, but it could be as a consequence of an acute exacerbation of the underlying disease and/or blockage of a major branch of the pancreatic duct by a protein plug, calculus or localized fibrosis [8]. The clinical presentation is quite variable and while some patients could be asymptomatic, others might present with abdominal catastrophe as a result of; bleeding, infection or rupture of the cyst $[9,10]$. In our study, 2 patients had acute hemorrhage into the cyst from erosion into the splenic artery while 1 patient had infection of the cyst and all 3 patients died. Various imaging modalities are used in the diagnosis of pancreatic pseudo-cysts with Computed Tomography (CT) being the gold standard with $82 \%$ to $100 \%$ sensitivity and 98\% specificity [11]. Endoscopic Ultrasound (EUS) has the highest sensitivity (93\% to $100 \%$ ) and specificity (92\% to $98 \%$ ) in differentiating acute fluid collections from pancreatic abscess and other pancreatic pseudocysts [12]. In our study 41 patients $(48.8 \%)$ of the pancreatic pseudocysts were associated with acute pancreatitis and 21 cases (25\%) were associated with chronic pancreatitis. However, it is worth mentioning that 8 cases of the 41 in the acute pancreatitis group had a background of chronic pancreatitis. 4 patients (19\%) were associated with pancreatic malignancy while 18 patients $(21.4 \%)$ were incidental finding on CT scans done for other reasons. These results are different from other studies showing the prevalence of pancreatic pseudocysts in acute pancreatitis to range from $6 \%$ to $18.5 \%[13,14]$. The prevalence of pancreatic pseudocysts in chronic pancreatitis is $20 \%-40 \%$ [15]. In our study, the most common underlying factor was alcoholic pancreatitis in 37 patients (44\%), gall stones in 19 patients (22.6\%), pancreatic malignancy in 4 patients $(4.8 \%)$, biliary strictures in 2 patients and trauma in 2 patients. Idiopathic pancreatic pseudocysts were seen in 20 patients (23.8\%), 18 of them were incidentally found on the CT scan. Of note, in 8 cases (40\%) of the idiopathic pseudo-cysts, were associated with non-pancreatic malignancies. Results from other series showed that pancreatic pseudocysts are most common in patients with chronic alcoholic pancreatitis (up to $78 \%$ ) [16], followed by idiopathic chronic pancreatitis (6\% to $16 \%$ ) and biliary pancreatitis (6\% to $8 \%$ ) [17]. The range of spontaneous regression of pancreatic pseudocysts ranges from $8 \%$ to $70 \%$ and the two major factors affecting this are; the size of the pseudocyst and the time since diagnosis [17]. In our study, 40 patients (47.6\%) had spontaneous resolution of their pseudocysts and only 4 patients had drainage procedure for their cysts (2 percutaneously and 2 endoscopically) in the initial period of the study and 1 further patient having endoscopic drainage in the follow up period of the 
study. The body of the pancreas was the most common site with 33 pseudo-cysts, followed by the head of the pancreas with 28 , tail 27 , uncinate process 4 , neck 2 , not specified 7, 1 in the junction between body and tail, and 2 in the junction between head and body. 9 patients in our study had multiple pseudocysts. The results in the literature about the site of pseudocysts within the pancreas is variable, as some studies showed that most pseudocysts would be in or near the tail of the pancreas [18]. In another study, most extra pancreatic pseudocysts were located in the body and tail region, whereas most intrapancreatic pseudocysts were in the head of the pancreas [19]. The overall mortality in our study was $50 \%$ (42 patients), however the cause specific mortality was only 9 patients (10.7\%). 59.5\% of the whole mortality cohort had alcoholic pancreatitis while $28.5 \%$ had gall stones pancreatitis. The 9 cause specific mortality patients; 7 were alcoholic pancreatitis and 2 were gall stones pancreatitis. On multivariate analysis there was a statistically significant association between the aetiology of pancreatitis and death which was also confirmed on Chi-square testing. There was no statistically significant association between the mode of pancreatitis (acute or chronic) and death. The duration of time form diagnosis of pancreatitis/ pancreatic pseudo-cyst to death was quite variable ranging from 8 to 3809 days (median 1018 days) in the whole mortality cohort. In the cause specific mortality group, the range was 10 to 1424 days (median 112 days), this was statistically significant.

\section{Conclusion}

Our study showed that alcoholic pancreatitis remains the most frequent underlying aetiology for pancreatic pseudocysts although it is not as common as in other studies. The incidence of incidental pseudocysts with no history of pancreatitis is higher than that in the literature. Around $50 \%$ of pseudo pancreatic cysts in our study resolved spontaneously; therefore, conservative treatment has a big role in management of pancreatic pseudocysts. Bleeding or infection of a pseudo pancreatic cyst is an emergency associated with high mortality and should be managed promptly with laparoscopic and endoscopic approaches now gaining popularity over the surgical approach which is only used if the previously mentioned approaches fail.

\section{References}

1. Khanna AK, Tiwary SK, Kumar P (2012) Pancreatic pseudocyst: Therapeutic dilemma. International Journal of Inflammation.

2. Bradley EL $3^{\text {rd }}$ (1993) A clinically based classification system for acute pancreatitis. Summary of the International Symposium on Acute
Pancreatitis, Atlanta, Ga, September 11 through 13, 1992. Arch Surg 128: 586-590.

3. Boerma D, Obertop H, Gouma DJ (2000) Pancreatic pseudocysts in chronic pancreatitis. Surgical or interventional drainage? Ann Ital Chir 71: 43-50.

4. Pitchumoni CS, Agarwal N (1999) Pancreatic pseudocysts. When and how should drainage be performed? Gastroenterol Clin North Am 28: 615-639.

5. Sandy JT, Taylor RH, Christensen RM, Scudamore C, Leckie P (1981) Pancreatic pseudocyst. Changing concepts in management. Am J Surg 141: 574-576.

6. Wade JW (1985) Twenty-five-year experience with pancreatic pseudocysts. Are we making progress? Am J Surg 149: 705-708.

7. Habashi S, Draganov PV (2009) Pancreatic pseudocyst. World J Gastrointest Endosc 15: 38-47.

8. Grace PA, Williamson RC (1993) Modern management of pancreatic pseudocysts. Br J Surg 80: 573-581.

9. Zdanyte E, Strupas K, Bubnys A, Stratilatovas E (2004) [Difficulties of differential diagnosis of pancreatic pseudocysts and cystic neoplasms] Medicina (Kaunas) 40: 1180-1188.

10. Gouyon B, Levy P, Ruszniewski P, Zins M, Hammel P, et al. (1997) Predictive factors in the outcome of pseudocysts complicating alcoholic chronic pancreatitis. Gut 41: 821-825.

11. Balthazar EJ, Freeny PC, Van Sonnenberg E (1994) Imaging and intervention in acute pancreatitis. Radiology 193: 297-306.

12. Lehman GA (1999) Pseudocysts. Gastrointest Endosc 49: 81-84.

13. Imrie CW, Buist LJ, Shearer MG (1988) Importance of cause in the outcome of pancreatic pseudocysts. Am J Surg 156: 159-162.

14. Maringhini A, Uomo G, Patti R, Rabitti P, Termini A, et al. (1999) Pseudocysts in acute nonalcoholic pancreatitis: incidence and natural history. Dig Dis Sci 44: 1669-1673.

15. Barthet M, Bugallo M, Moreira LS, Bastid C, Sastre B, et al. (1993) Management of cysts and pseudocysts complicating chronic pancreatitis. A retrospective study of 143 patients. Gastroenterol Clin Biol 17: 270276.

16. Ammann RW, Akovbiantz A, Largiader F, Schueler G (1984) Course and outcome of chronic pancreatitis. Longitudinal study of a mixed medicalsurgical series of 245 patients. Gastroenterology 86: 820-828.

17. Markus M Lerch, Albrecht Stier, Ulrich Wahnschaffe, Julia Mayerle (2009) Pancreatic Pseudocysts: Observation, Endoscopic Drainage, or Resection? Dtsch Arztebl Int 106(38): 614-621.

18. Bourliere M, Sarles H (1989) Pancreatic cysts and pseudocysts associated with acute and chronic pancreatitis. Dig Dis Sci 34: 343-348.

19. Kloppel G, Maillet B (1991) Pseudocysts in chronic pancreatitis: a morphological analysis of 57 resection specimens and 9 autopsy pancreata. Pancreas 6: 266-274.

20. Bradley EL, Gonzalez AC, Clements JL (1976) Acute pancreatic pseudocysts: incidence and implications. Jr Ann Surg 184(6): 734-737. 
(C) (1) This work is licensed under Creative

To Submit Your Artic

DOI: $10.32474 /$ SCSOAJ.2019.03.000152

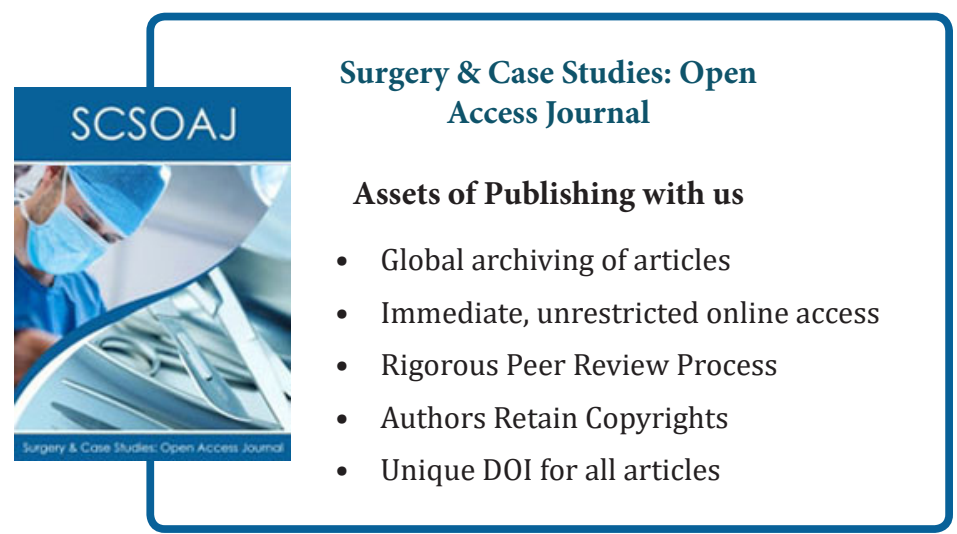

